\title{
Surgical resection of a giant cardiac hemangioma encroached upon the right coronary artery: A case report
}

guangpu fan ${ }^{1}$ and $\mathrm{Yu} \mathrm{Chen}^{1}$

${ }^{1}$ Peking University People's Hospital

December 6, 2020

\begin{abstract}
Cardiac hemangioma is relatively rare for primary cardiac tumors. The diagnose is mainly based on surgical resection and biopsy, imaging examinations only provide limited diagnostic clues. For those giant cardiac hemangiomas, which may raise a risk of rupture, need thoroughly surgical removal. However, meticulous follow-up is required due to its possibility of recurrence.

Surgical resection of a giant cardiac hemangioma encroached upon the right coronary artery: A case report Guangpu Fan, PhD. , Yu Chen, MD.
\end{abstract}

Authors:

Guangpu Fan, Ph.D.

Peking University People's Hospital, cardiac surgery. Peking, China.

Yu Chen, MD.

Peking University People's Hospital, cardiac surgery. Peking, China.

*Corresponding author:

Yu Chen, MD.

Peking University People's Hospital, cardiac surgery. Peking, China. 100044. Tel: 010-88324550. Email: micsc@sina.com

Data availability statement:

The data used to support the findings of this study are available from the corresponding author upon request.

Funding:

None

Conflict of interest:

None.

IRB approval, consent statement and clinical trial registration:

N/A

Abstract

Background 
Cardiac hemangioma is relatively rare for primary cardiac tumors. The diagnose is mainly based on surgical resection and biopsy, imaging examinations only provide limited diagnostic clues. For those giant cardiac hemangiomas, which may raise a risk of rupture, need thoroughly surgical removal. However, meticulous follow-up is required due to its possibility of recurrence.

Case presentation

A 62-year-old woman presented with symptoms of respiratory distress and exercise dyspnea for two years. 8 months ago, the chest CT scan revealed an occupation in anterior mediastinum, therefore she was admitted to thoracic surgery department with an impression diagnosis of invasive thymoma.

No myasthenia gravis or dysphagia was noted at her admission, blood test for tumor markers was negative, the respiratory and cardiac portions of the physical examination had no abnormalities. Thoracic enhanced CT scan surprisingly revealed the mass was closely related to the right coronary artery(RCA). Therefore, the patient was referred to cardiac surgery department, and a detailed examination was arranged (Fig 1, A-F): Coronary CT angiography confirmed the RCA was surrounded by a giant mass, with inner irregular enhancement. PET-CT indicated a slightly increase of the FDG metabolism. Coronary arteriography showed a smooth shape of the RCA, with small branches stretched into the mass.

A "surgical resection" plan was made by the cardio-thoracic team. During surgery, a median sternotomy approach was used, the mass was confirmed to be in the pericardial cavity, other than thymus (Fig 2A). The giant reddish and smooth mass was attached to the right ventricle (RV), the border between the mass and RV was fuzzy, and a groove implied the RCA's trace. Cavernous hemangioma was considered by visual judgement and frozen pathological examination. After cardiopulmonary bypass was established with bicaval cannulation, the cardioplegic arrest was obtained. The tumor was carefully and maximally resected, and a pericardium patch was used to reconstruct the RV myocardium (Fig 2B). The treatment of RCA was based on the concept of "reservation", the wrapped RCA was fully preserved, intraoperative Transit Time Flow Measurement confirmed a satisfactory flow (Fig 2C). The postoperative course was uneventful, and the patient was discharged with no complications.

Pathological examination demonstrated amounts of vascular structures, stained vascular endothelial cells, which was categorized as a cavernous hemangioma $(8.5 \mathrm{~cm} \times 6.0 \mathrm{~cm} \times 3.0 \mathrm{~cm})($ Fig $2 \mathrm{D}-\mathrm{H})$, immunohistochemical analysis showed positive CD31, CD34, ERG staining.

\section{Discussion}

It is relatively rare for primary cardiac tumors, of cardiac hemangiomas, is amount for $5 \%-10 \%$ of the benign neoplasms [1]. Whether these benign tumors need surgical treatment is still controversial, however, due to the location, size of the lesions are various, the surgical indications may raise for the conspicuousness of clinical symptoms. Cardiac hemangiomas may occur in the epicardium, myocardium, or endocardium, and all cardiac chambers. Presentations include arrhythmias, conduction disturbances, congestive heart failure, coronary insufficiency, chest pain, cough, and dysphagia [2].

Based on the morphologic pattern of their vascular channels, endothelial cells, and supporting stroma, cardiac hemangiomas can be classified into three groups: capillary, arteriovenous, and cavernous hemangiomas [3]. Imaging examination, including echocardiography, computed tomography, or magnetic resonance imaging, only provides limited diagnostic clues. In this case, enhanced CT indicated irregular vascular enhancement, and angiography indicated collateral vessels, which are helpful for diagnosis.

Most of the results of surgical removal are satisfied, but there are still cases that cannot be excised $[4,5]$. The decision to operate on this giant tumor was based on the risk of rupture, the presence of symptoms, and the inconsistencies in tumor location across imaging modalities. Although the tumor often adheres to surrounding tissues, it could be separated finely. In this case of a giant cardiac hemangioma, with the RCA being wrapped, the operation difficulty was evident raised. Fully preservation of the RCA, thus avoiding coronary bypass grafting was considered as a highlight of the operation. Because of the possibility of recurrence, fully resection 
is quite necessary, but attention should be paid to minimize the damage of ventricular muscle to avoid interrupting cardiac function.

Conclusion

In conclusion, we reported the surgical resection of a giant cardiac hemangioma with the right coronary artery being wrapped under cardiopulmonary bypass. Although the definitive diagnosis of primary cardiac tumors is difficult, the postoperative CT angiography in this patient before discharge showed thorough remove of the tumor and sufficient reservation of the RCA. Meticulous follow-up is required for this benign tumor after the resection to beware of recurrence.

Conflict of interest:

None.

References

1.Jain D, Maleszewski JJ, Halushka MK. Benign cardiac tumors and tumorlike conditions. Ann Diagn Pathol 2010;14:215-30.

2.C. Eftychiou, L. AntoniadesCardiac hemangioma in the left ventricle and brief review of the literatureJ Cardiovasc Med, 10 (2009), pp. 565-567

3.Grebenc ML, Rosado de Christenson ML, Burke AP, Green CE, Galvin JR. Primary cardiac and pericardial neoplasms: radiologic-pathologic correlation. Radiographics 2000;20:1073-103.

4. Li W, Teng P, Xu H, Ma L, Ni Y. Cardiac Hemangioma: A Comprehensive Analysis of 200 Cases. Ann Thorac Surg. 2015 Jun;99(6):2246-52.

5. Botha J, Ihlberg L, Elhenawy A, Abbott M, Butany J, Paul N, Brister SJ. A giant cavernous hemangioma of the heart. Ann Thorac Surg. 2010 Jul;90(1):293-5.

Figure 1. Images of the preoperative examination. Coronary CT angiography (A-C) confirmed the RCA was surrounded by an $8.4 \mathrm{~cm} \times 4.5 \mathrm{~cm} \times 3.0 \mathrm{~cm}$ mass (yellow asterisk). Irregular vascular enhancement could be seen. Three-dimensional reconstruction showed the right ventricle was oppressed (yellow arrow). PET-CT (E) indicated a slightly increase of the FDG metabolism. Coronary arteriography (F) showed an abnormally originated of the right coronary artery (RCA, white arrow) from the left coronary sinus, left main (LM, white arrow) could be seen, small branches stretched into the mass from the coronary artery was demonstrated (yellow asterisk).

Figure 2. The reddish and smooth tumor was located pericardium (A, yellow asterisk), the border between the mass and RV was fuzzily along the outflow tract (A, dotted line). After resection of the tumor, the RCA was totally reserved (B, yellow dotted line), and a pericardium patch was used to reconstruct the injured RV myocardium (B). TTFM show a satisfied flow of the original RCA, with $81 \mathrm{ml} / \mathrm{min}$ of blood flow, and 0.6 pulsatility index (PI) (C). Pathological examination categorized a cavernous hemangioma $(8.5 \mathrm{~cm} \times 6.0 \mathrm{~cm} \times 3.0 \mathrm{~cm})(\mathrm{D}$, yellow asterisk $)$, the trace of RCA was showed at this anterior of the gross specimen $(\mathrm{D}$, yellow dotted line), hematoxylin and eosin staining $(\times 200)$ showing large vessels with a considerable amount of blood enclosed within the vascular endothelium (E), with immunohistochemical analysis $(\times 200)$ of positive CD31, CD34, ERG staining (F-H). 

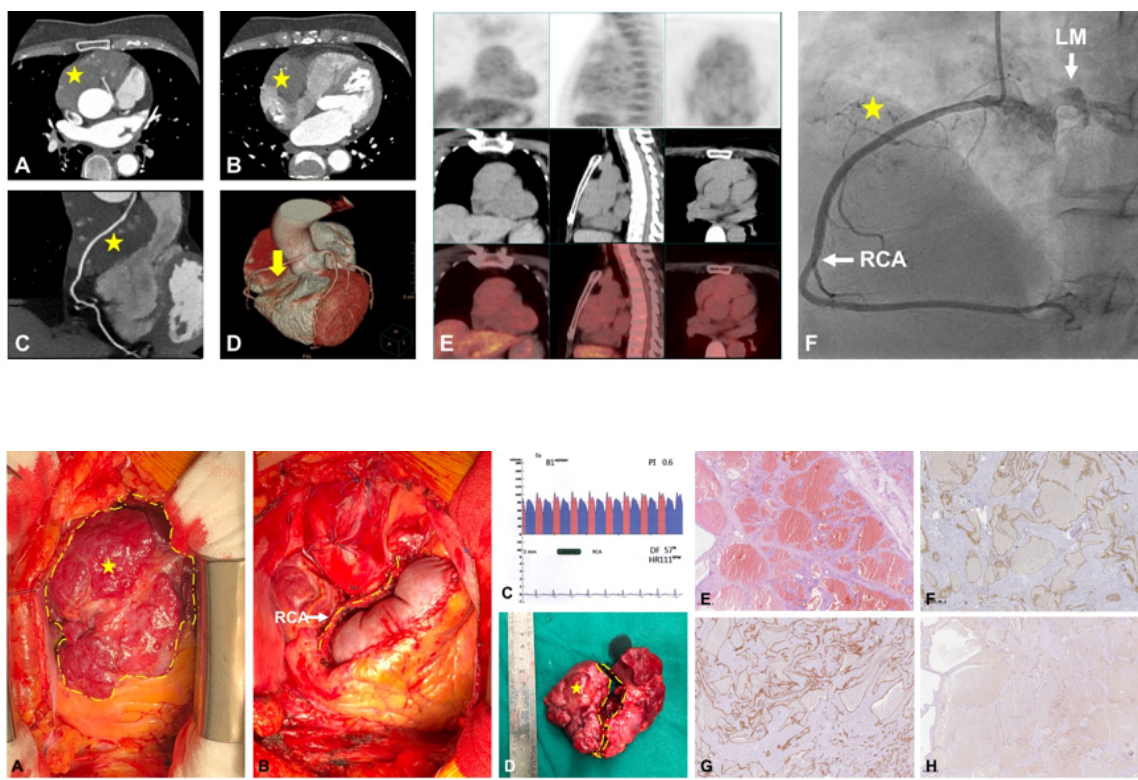